\title{
The impact of blood group on survival following critical illness
}

\section{Slade, $\mathbf{R}^{\mathbf{1}}$., Alikhan, $\mathbf{R}^{\mathbf{2}}$., Wise, MP1., Germain, $\mathbf{L}^{\mathbf{1}}$., Morgan, MPG'}

University Hospital of Wales Critical Care Directorate' ${ }^{1}$ Department of Haematology ${ }^{2}$

\section{Introduction}

Predicting patient outcomes following critical illness is challenging. Although genetic variation is known to be one factor in determining survival in intensive care, it is difficult to measure in clinical practice (1). Recent evidence has suggested that patients with blood group $A B$ are more likely to survive following major cardiac surgery and this is associated with a reduced number of blood transfusions (2). There is a body of evidence which suggests that blood group $A B$ has higher levels of Von-Willebrand factor and factor VIII $(3,4)$, making this group more pro-thrombotic, which may be beneficial in patient groups where bleeding risks are high. However, there is no current data to indicate whether a patient's blood group affects general intensive care outcomes.

\section{Objectives}

The objective of the study was to determine if $A B O$ blood group affects survival in intensive care. The primary outcome measure was 90-day mortality with a secondary outcome measure of the percentage of patients receiving a blood transfusion.

\section{Materials and Methods}

We performed a retrospective cohort analysis of all patients admitted to the Intensive Care Unit of a major tertiary University Hospital between 2006 and 2016. Baseline characteristics for each blood group were collected including, age, gender, BMI, surgery during admission, days in hospital, ethnicity and APACHE II score. 90-day survival was estimated by Kaplan-Meier plots. ABO blood groups were individually compared and then pooled to compare $A B$ vs non-AB blood groups.

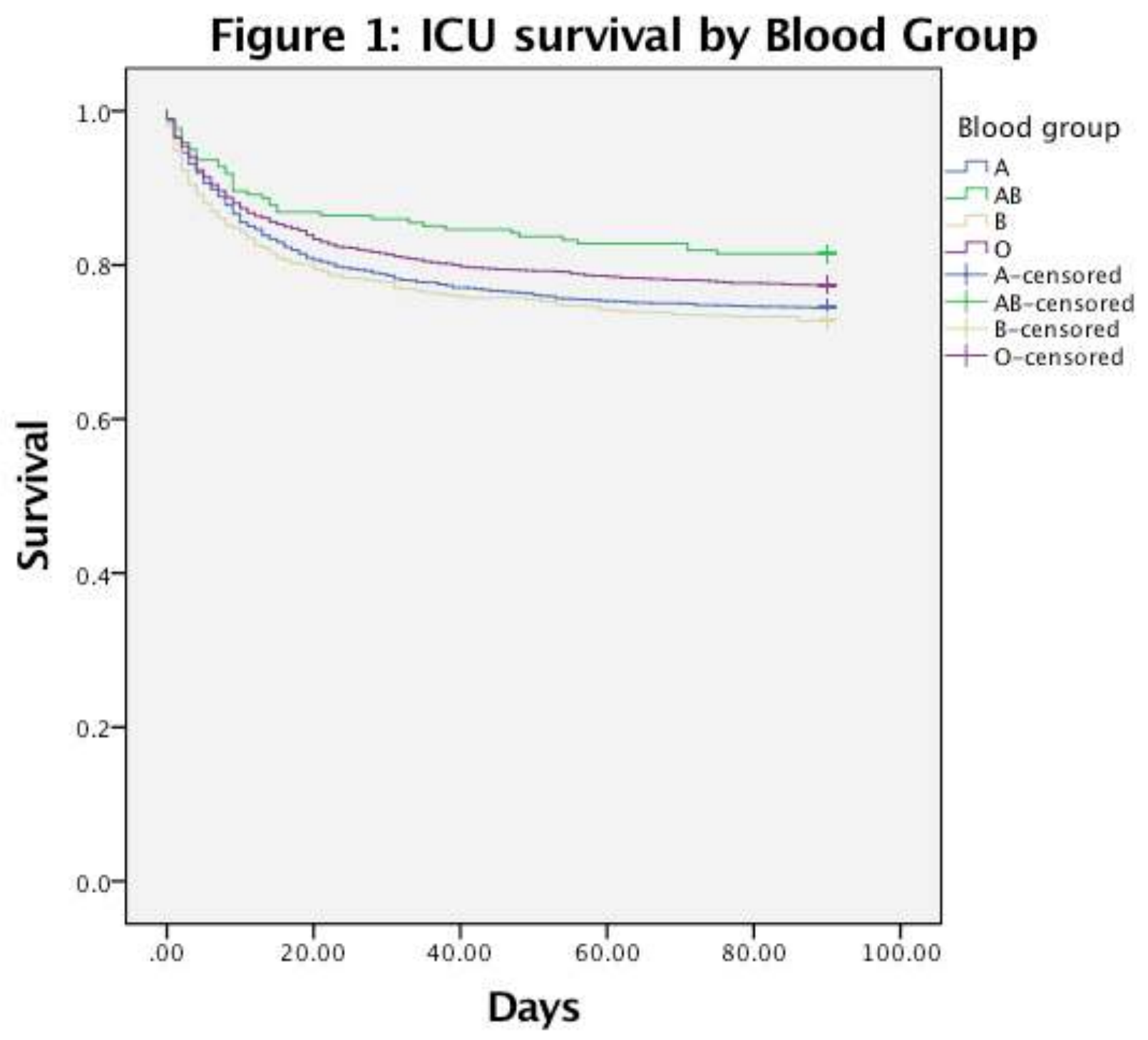

Maslove DM, Lamontagne F, Marshall JC, Heyland DK. A path to precision in the ICU. Crit Care. 2017;21(1):79 Welsby IJ, Phillips-Bute B, Mathew JP, Newman MF, Becker R, Rao S, et al. ABO blood group influences transfusion and survival after cardiac surgery. J Thromb Thrombolysis. 2014 Oct 17;38(3):402-8

3. Franchini M, Capra F, Targher G, Montagnana M, Lippi G. Relationship between ABO blood group and vor Willebrand factor levels: from biology to clinical implications. Thromb J. BioMed Central; 2007 Sep 25;5:14. 4. Song J, Chen F, Campos M, Bolgiano D, Houck K, Chambless LE, et al. Quantitative Influence of ABO Blood Group on Factor VIII and Its Ratio to von Willebrand Factor, Novel Observations from an ARIC Study of 11,673 Subjects. Miyata T, editor. PLoS One. Public Library of Science; 2015 Aug 5;10(8):e0132626 5. Firkin F, Chesterman C, Pennington D, Rush B. De Gruchy's Clinical haematology in medical Practice. 5th ed.
Figure 2: ICU survival blood group $A B$ vs non- $A B$

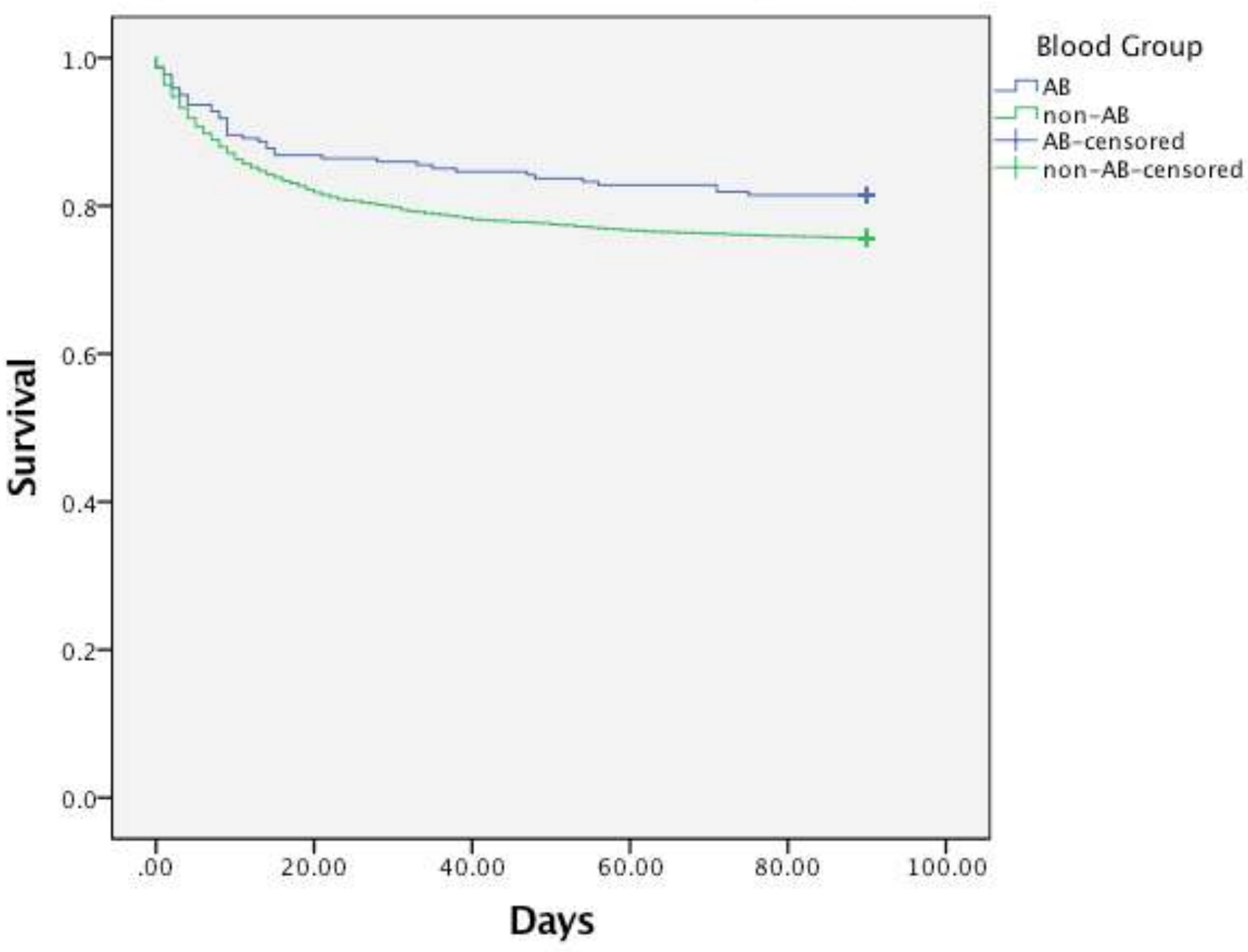

\section{Results}

7340 patients were included in the study. Blood group $A B$ accounted for $3 \%$ (221), A 41\% (3008), B 10.6\% (775) and O $45.4 \%$ (3336). These values are similar to UK averages (5).

Blood group $A B$ had the greatest survival benefit when assessing per individual blood group Figure 1 (blood group $A B$ 90-day survival estimate $76.75,95 \% \mathrm{Cl} 72.89-80.61$ with the overall estimate 72.07, 95\% Cl 71.31-72.82) (Log rank Chi square 16.128, $p=0.001)$. Transfusion requirements were similar in all groups with no significant difference between the percentages of patients transfused (AB 23.1\%, A 21.5\%, B 18.7\%, O19.9\%, Pearson chi square $5.060 \mathrm{p}=0.167)$.

When pooling blood group data, Kaplan-Meir analysis showed blood group $A B(n=221)$ to have improved survival compared to non-AB blood groups Figure 2 ( $n=7119)$ (blood group AB 90-day survival estimate $76.75,95 \% \mathrm{Cl} 72.89-80.61$ non-AB groups 71.92, 95\% Cl 71.15-72.69) (log rank Chi Square 3.890, $p=0.049)$.

\section{Conclusion}

- Intensive care patients with blood group $A B$ have a higher 90day survival compared with other blood groups.

- There was no correlation between blood group and percentage of patients receiving transfusion. Blood group $A B$ is relatively rare accounting for $3 \%$ of the study population and therefore our study may have been underpowered to determine this bleeding risk.

- Improved survival outcomes in blood group $A B$ are a promising area for further study including any mechanistic basis for this finding. 